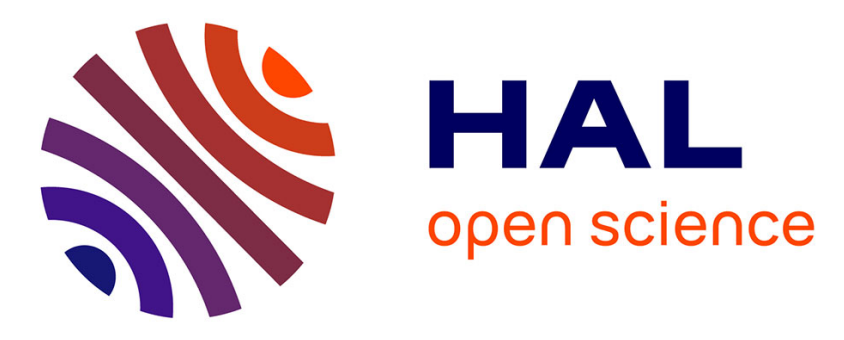

\title{
Simulation and Analysis of the Spectroscopic Properties of Oxyluciferin and Its Analogues in Water
}

\author{
Cristina Garcia-Iriepa, Pauline Gosset, Romain Berraud-Pache, Madjid \\ Zemmouche, Gregory Taupier, Kokou Dodzi Dorkenoo, Pascal Didier, Jérémie \\ Léonard, Nicolas Ferré, Isabelle Navizet
}

\section{To cite this version:}

Cristina Garcia-Iriepa, Pauline Gosset, Romain Berraud-Pache, Madjid Zemmouche, Gregory Taupier, et al.. Simulation and Analysis of the Spectroscopic Properties of Oxyluciferin and Its Analogues in Water. Journal of Chemical Theory and Computation, 2018, 14 (4), pp.2117 - 2126. 10.1021/acs.jctc.7b01240 . hal-01769577

\section{HAL Id: hal-01769577 \\ https://hal.science/hal-01769577}

Submitted on 1 Mar 2019

HAL is a multi-disciplinary open access archive for the deposit and dissemination of scientific research documents, whether they are published or not. The documents may come from teaching and research institutions in France or abroad, or from public or private research centers.
L'archive ouverte pluridisciplinaire HAL, est destinée au dépôt et à la diffusion de documents scientifiques de niveau recherche, publiés ou non, émanant des établissements d'enseignement et de recherche français ou étrangers, des laboratoires publics ou privés. 


\section{Simulation and analysis of the spectroscopic}

\section{properties of oxyluciferin and its analogues in water.}

Cristina García-Iriepa, ${ }^{* a}$ Pauline Gosset, ${ }^{b}$ Romain Berraud-Pache, ${ }^{a}$ Madjid Zemmouche, ${ }^{a}$ Grégory Taupier, ${ }^{b}$ Kokou Dodzi Dorkenoo, ${ }^{b}$ Pascal Didier, ${ }^{c}$ Jérémie Léonard ${ }^{b}$, Nicolas Ferréd and Isabelle Navizet*a

${ }^{a}$ Université Paris-Est, Laboratoire Modélisation et Simulation Multi Echelle, MSME, UMR 8208 CNRS, UPEM, 5 bd Descartes, 77454 Marne-la-Vallée, France

${ }^{\mathrm{b}}$ Université de Strasbourg, CNRS, Institut de Physique et Chimie des Matériaux de Strasbourg and Labex NIE, 67000 Strasbourg, France

c Université de Strasbourg, CNRS, Laboratoire de Biophotonique et Pharmacologie UMR 7213, 67000 Strasbourg, France

d Aix-Marseille Univ., CNRS, Institut de Chimie Radicalaire, 13013 Marseille, France

KEYWORDS. Oxyluciferin, TD-DFT, Molecular Dynamics, QM/MM, Absorption Spectra, Emission Spectra, Bioluminescence.

ABSTRACT. Firefly bioluminescence is a quite efficient process largely used for numerous applications. However, some fundamental photochemical properties of the light emitter are still to be analyzed. Indeed, the light emitter, oxyluciferin, can be in six different forms due to inter- 
exchange reactions. In this work, we present the simulation of the absorption and emission spectra of the possible natural oxyluciferin forms in water and some of their analogues considering both, the solvent/oxyluciferin interactions and the dynamical effects by using MD simulations and QM/MM methods. On the one hand, the absorption band shapes have been rationalized by analyzing the electronic nature of the transitions involved. On the other hand, the simulated and experimental emission spectra have been compared. In this case, an ultrafast excited state proton transfer (ESPT) occurs in oxyluciferin and its analogues, which impairs the detection of the emission from the protonated state by steady-state fluorescence spectroscopy. Transient absorption spectroscopy was used to evidence this ultrafast ESPT, and rationalize the comparison between simulated and experimental steady-state emission spectra. Finally, this work shows the suitability of the studied oxyluciferin analogues to mimic the corresponding natural forms in water solution, as an elegant way to block the desired inter-exchange reactions allowing the study of each oxyluciferin form separately.

\section{INTRODUCTION}

Bioluminescence is the process responsible for flashes of bright light coming out from a wide variety of living organisms. It results from the conversion of chemical energy into visible light, catalyzed by a protein. This natural phenomenon is present in several organisms such as bacteria, beetles, squid, worms, fungi, algae and jellyfish. ${ }^{1,2}$ In the last decades, the study of the bioluminescence mechanism as well as the spectroscopic properties of the emitter species have attracted considerable interest among scientists. In this regard, the bioluminescent process of the jellyfish (aequorin/green fluorescent protein system) $)^{3-6}$ and the one of fireflies ${ }^{7,8}$ are the most studied systems up to now. 
In particular, its large number of applications has motivated the extensive study of firefly bioluminescence. For instance, firefly bioluminescence has been used for rapid analysis of bacterial contamination, ${ }^{9}$ in vivo bioluminescence imaging, ${ }^{10,11}$ multiple gene expression and regulation, ${ }^{12,13}$ monitoring of cell dynamics ${ }^{14,15}$ and drug screening ${ }^{16}$ among others. Nowadays, the general bioluminescent mechanism in fireflies is well accepted and consists in an enzymatic multistep oxidation. ${ }^{17-19}$ First, an enzyme called luciferase binds a substrate known as luciferin and catalyzes the formation of luciferyl adenosine monophosphate. Afterwards, the emitter species, so-called oxyluciferin $\left(\mathrm{OxyLH}_{2}\right)$, is generated in the first singlet-excited state by decomposition of a cyclic peroxide and release of $\mathrm{CO}_{2}$. Finally, oxyluciferin decays to the ground state by emitting light in the visible spectral window.

It is remarkable that for fireflies, the color emission can vary from yellow-green to red depending on diverse factors such as $\mathrm{pH},{ }^{20}$ the presence of bivalent metal ions, ${ }^{21}$ temperature, ${ }^{22}$ luciferase mutations ${ }^{23}$ and luciferin structure modifications. ${ }^{24,25}$ However, despite the large number of studies focused on understanding the origin of the emission color modulation, this mechanism remains unsolved. ${ }^{26-28}$ Indeed, the fact that oxyluciferin can exist in six different chemical forms further complicates the rationalization of the emission color modulation. These six different chemical forms of oxyluciferin rise from a triple equilibrium of phenol and/or enol deprotonation together with a keto/enol tautomerization (Chart 1), being these equilibria sensitive to the solvent, $\mathrm{pH}$ or interactions with bases. ${ }^{29-31}$ 


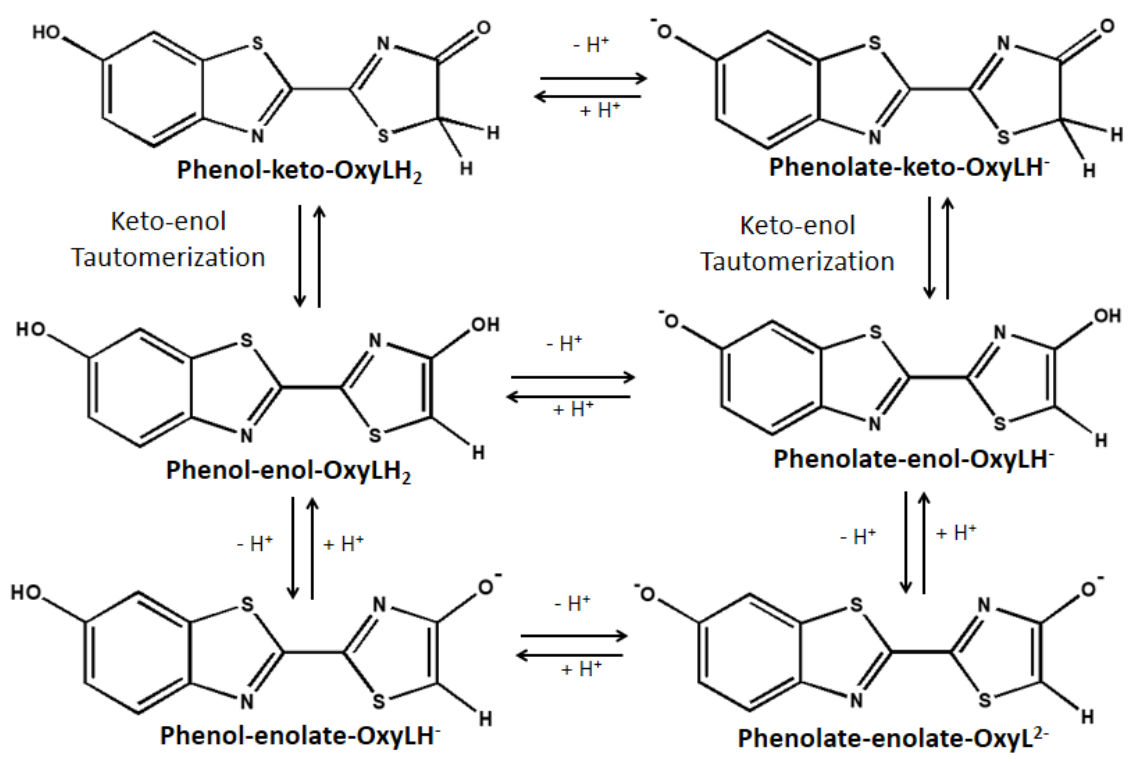

Chart 1. Chemical equilibria leading to the six possible oxyluciferin forms.

In order to simplify this scenario, some studies have proposed novel derivatives as an elegant way to block one or more of these chemical equilibria. For instance, the 5,5-dimethyloxyluciferin analogue has been used to block the keto/enol tautomerization ${ }^{27,31-33}$ but, it is unstable upon photoexcitation. ${ }^{34}$ For this reason, the 5,5-spirocyclopropyloxyluciferin (5,5-CprOxyLH in Chart 2) analogue has emerged as a best candidate to mimic the keto form of oxyluciferin. ${ }^{34,35}$ Moreover, other oxyluciferin derivatives have been proposed in order to block the deprotonation/protonation equilibrium of the phenol and/or enol groups such as the 4-methoxyoxyluciferin (4-MeOxyLH in Chart 2) or 6'-methoxyoxyluciferin (6'-MeOxyLH in Chart 2). ${ }^{27,33-35}$ This strategy enables the experimental study of the individual chemical forms of oxyluciferin that can otherwise not be isolated.

A different approach that can be followed to investigate the photochemical properties of the less stable oxyluciferin forms is to perform computational studies. In the past, computational studies have provided essential information about the bioluminescence mechanism of fireflies, ${ }^{36-38}$ 
emission color modulation, ${ }^{28,39}$ relative stability of the different oxyluciferin forms ${ }^{40}$ as well as their pKa values. ${ }^{41,42}$ This way, computational studies have become essential to learn more about this system.

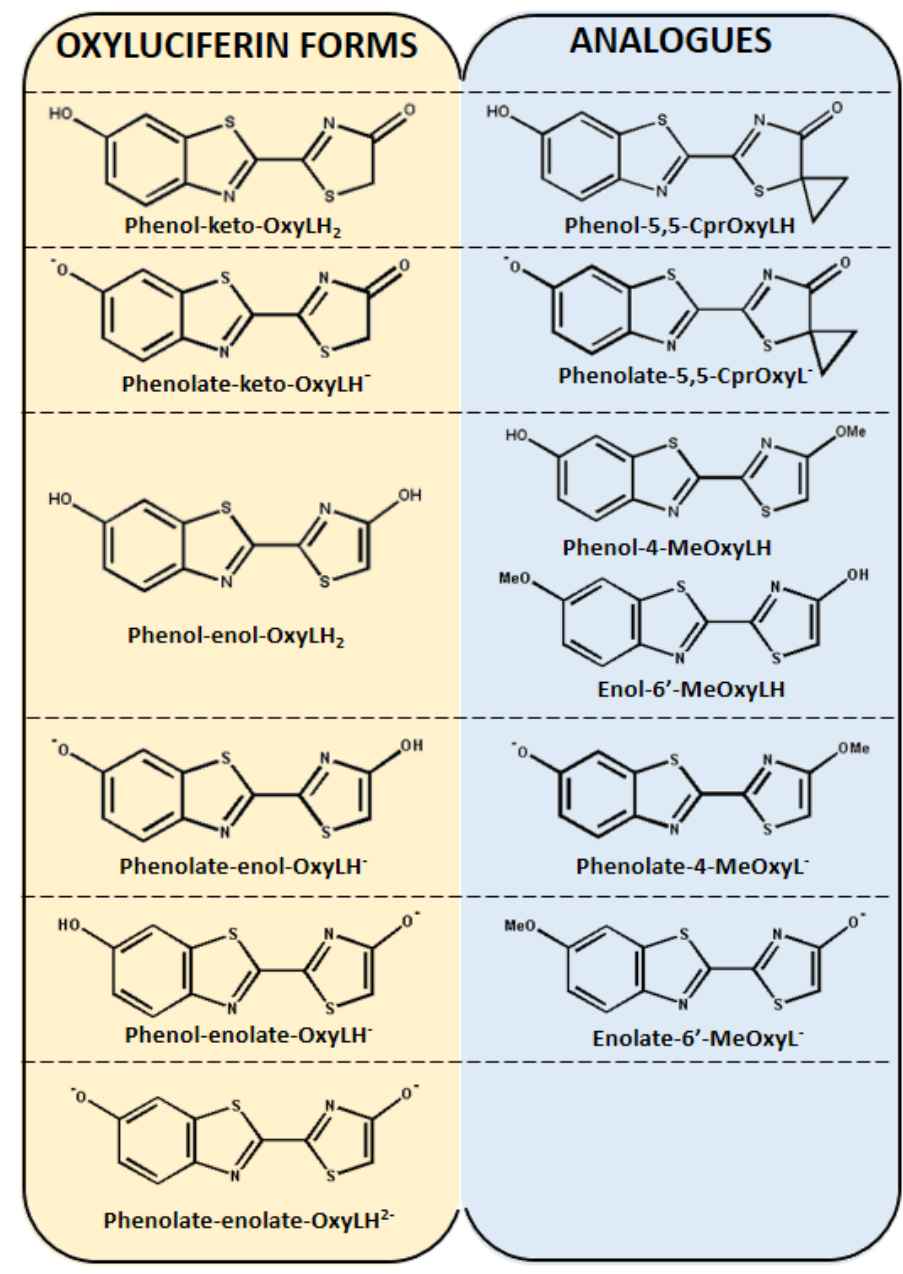

Chart 2. Chemical structures of the six possible chemical forms of oxyluciferin and the corresponding analogues under study.

Here, we propose a simple approach to simulate the absorption and emission spectra of all possible oxyluciferin forms and some analogues in explicit water solution. For this aim, molecular dynamics have been performed to consider the explicit water molecules and QM/MM methods have been used to compute the excitation and emission energies. More precisely, this work 
presents three novelties compared to the computational studies reported up to now. First, the absorption and emission spectra have been simulated considering both the dynamical effect and the explicit interactions between the solvent and oxyluciferin by means of molecular dynamics. So far, the excitation and emission energies of some natural oxyluciferin forms have been computed in the gas phase, ${ }^{36,43}$ considering the solvent implicitly with the polarizable continuum model $(\mathrm{PCM}),{ }^{44,45}$ by microsolvation including 11 water molecules ${ }^{46}$ or using a canonical ensemble of hydration structures obtained from first-principles molecular dynamics. ${ }^{47}$

Second, this work presents for the first time a detailed analysis of the electronic nature of the vertical transitions not only to the first singlet excited state but also to upper-lying excited states. This data is important to rationalize the shape of the absorption spectra, in some cases structured, and to reveal hidden transitions from an experimental point of view (e.g. embedded within a more intense and broad band). Third, a quite extensive study of the spectral properties of oxyluciferin and its analogues is reported as it covers a total of 12 derivatives: the six possible chemical forms of oxyluciferin and three analogues, considering the protonated and the deprotonated forms (Chart 2). Moreover, a comparative study of oxyluciferin and its analogues is presented to validate if they can be used to mimic natural oxyluciferin in water solution. It is remarkable that in all cases, a good agreement between the experimental and simulated spectra has been found using the strategy proposed in this study.

\section{METHODS}

\section{Computational Methods.}

Classical dynamic simulations have been performed with Amber $14^{48}$ in order to obtain a statistical number of snapshots to perform Quantum Mechanics/Molecular Mechanics (QM/MM) 
calculations to simulate the absorption (emission) spectra considering explicit water molecules. For this aim, the structure of the compound optimized in the ground (excited) state has been selected as the starting point for MD simulations. The bond distances, angles, dihedrals and charges parameters for the oxyluciferin derivatives (geometry and charges of the minima in the ground and first excited states) have been derived from the QM calculations in gas phase and used as the starting point. The compound has been solvated with TIP3P water molecules ${ }^{49}$ within an octahedral box, ensuring a solvent shell of at least $10 \AA$ around the molecule, corresponding to around 900 water molecules. Then, the system has been minimized, followed by a heating step from $100 \mathrm{~K}$ to $300 \mathrm{~K}$ in $20 \mathrm{ps}$. Afterwards, a production run of $10 \mathrm{~ns}$ has been performed with a 2 fs time step, under NPT conditions ( $300 \mathrm{~K}$ and $1 \mathrm{~atm}$ ) and using periodic boundary conditions. The pressure and temperature were assured by the Berendsen algorithm. ${ }^{50}$

Then, 100 snapshots have been extracted and the excitation (emission) energies computed at the QM/MM level of theory, being enough to achieve the spectral shape convergence (Figure S2 and S7). At least 7 electronic excited states have been considered for the excitation energy calculation to simulate the spectra up to $300 \mathrm{~nm}$. In all cases, the absorption and emission spectra have been simulated as a convolution of the excitation and emission energies of 100 snapshots (full-width at half-maximum of $0.2 \mathrm{eV}$ ). The parameters have been updated as many times as needed by optimizing the system (derivative and explicit water molecules) at the $\mathrm{QM} / \mathrm{MM}$ level to reach the spectral shape convergence of the simulated spectra. In particular, for each set of parameters, a corresponding $10 \mathrm{~ns}$ MD was performed, 100 snapshots were extracted and the QM/MM absorption (emission) spectra simulated. New parameters were adjusted from the QM/MM calculation and the process was performed with the new set of parameters until reaching the spectral shape convergence between the previous and the next simulated spectra (Figure S1). In 
order to further check the suitability of the oxyluciferin parameters we have compared the absorption and emission spectra simulated with and without optimizing oxyluciferin at the QM/MM level of theory. In all cases, similar spectra have been obtained (Figure S3 and S6).

For the QM/MM calculations, the water molecules were treated at the MM level whereas the oxyluciferin derivative was included in the QM region and its excitation (emission) energy computed at the B3LYP $51-53 / 6-311 \mathrm{~g}(2 \mathrm{~d}, \mathrm{p})$ level of theory. This functional and basis set have been selected as they have been previously reported to be suitable for this chromophore. ${ }^{47,54-56}$ Calculations in gas phase have been performed using Gaussin $09^{57}$ and the functional and basis set used for the QM/MM calculations. The QM/MM calculations have been carried out using a QM/MM coupling scheme ${ }^{58}$ between Gaussian $09^{57}$ and Tinker. ${ }^{59}$ In particular, the interaction between the QM charge density (electrons and nuclei) and the external electrostatic potential of the MM part was computed by the electrostatic potential fitted (ESPF) method. ${ }^{58}$ The microiterations technique ${ }^{60}$ was used to converge the MM subsystem geometry for every QM minimization step.

\section{Experimental Methods.}

The model analogues 4-MeOxyLH and 6'-MeOxyLH were synthesized as previously reported. ${ }^{33}$ The synthesis and characterization of the new spirocyclic analogues 5,5-CprOxyLH will be reported elsewhere. Stock solutions of all compounds were prepared in spectroscopic grade DMSO (Sigma-Aldrich) and stored in several aliquots at $-20{ }^{\circ} \mathrm{C}$ to minimize the freeze-thaw cycle. They were further diluted 1000-fold to a final concentration of about a few $\mu \mathrm{M}$ in aqueous buffer with different $\mathrm{pH}$. The buffers were prepared by diluting a $1 \mathrm{M}$ stock solution in deionized, Milliporepurified water (18.2 M $\Omega$ ). Buffered stock solutions were prepared as follows: $75 \mathrm{mM} \mathrm{NaCl} / 20$ 
$\mathrm{mM} \mathrm{KH} \mathrm{PO}_{4} / 0.2 \mathrm{mM} \mathrm{MgCl}_{2}$ for $\mathrm{pH} \leq 7.0$ and $75 \mathrm{mM} \mathrm{NaCl} / 25 \mathrm{mM}$ Tris(hydroxymethyl)aminomethane (TRIS) $/ 0.2 \mathrm{mM} \mathrm{MgCl}_{2}$ for $\mathrm{pH}>7.0$. The buffer was separated into several fractions, and their $\mathrm{pH}$ was adjusted by $250 \mathrm{mM} \mathrm{HCl}$ or by $250 \mathrm{mM} \mathrm{NaOH}$ separately at $20{ }^{\circ} \mathrm{C}$. Separate buffers at different $\mathrm{pH}$ were used in all spectral measurements.

Absorption spectra were recorded with a Cary-4000 spectrometer (Agilent Technologies). Steadystate fluorescence spectra were recorded with Fluoromax 4 spectro-fluorometer (Horiba Jobin Yvon) with $2 \mathrm{~nm}$ excitation/emission slit and corrected for the instrumental response characteristics.

The femtosecond source is a regenerative amplifier that delivers $120 \mathrm{fs}$ pulses at $800 \mathrm{~nm}$ with a repetition rate of 5kHz ("Spitfire", Spectra-Physics, Mountain View, CA, USA). We generated $400 \mathrm{~nm}, 150$ fs pump pulse by second harmonic generation in a BBO crystal. We obtained supercontinuum probe pulse $(430 \mathrm{~nm}$ to $700 \mathrm{~nm}$ ) by self phase modulation in a $2 \mathrm{~mm}$ thick sapphire crystal. Pump and probe beams were focused by the same $38.1 \mathrm{~mm}$ focal length off-axis parabolic mirror on a $1 \mathrm{~mm}$ thick quartz cuvette containing the sample. To excite a fresh volume of the sample for each pump pulse, the cuvette was moved continuously. Time and spectrally resolved differential transmission $\Delta \mathrm{T} / \mathrm{T}(\tau, \lambda)$ of the probe as a function of the pump-probe delay $\tau$ and the wavelength $\lambda$ was measured in a standard pump-probe configuration. The spectra were obtained by dispersing the transmitted reference and probe beams in a spectrometer followed by a CCD detector (Roper, Trenton, NJ, USA). The analogues concentration was adjusted to obtain an optical density of 0.1 per $\mathrm{mm}$ at $400 \mathrm{~nm}(\sim 50 \mu \mathrm{M})$ to optimize the signal to noise ratio of the differential transmission of the probe. 


\section{RESULTS AND DISCUSSION}

Here is presented a detailed analysis of the photochemical and photophysical properties of all the possible chemical forms of oxyluciferin and their analogues in explicit water (Chart 2). For this study we have selected the following oxyluciferin analogues: $i$ ) 5,5-CprOxyLH which presents a cyclopropyl substituent in the thiazole ring to block the keto-enol tautomerization; ii) 4-MeOxyLH which has a methoxy group in the enol moiety of the thiazole ring to block both the keto-enol tautomerization and enol deprotonation and iii) 6'-MeOxyLH that presents a methoxy group in the phenol moiety to block the phenol deprotonation. In particular, this section is divided into three parts: i) Analysis of the possible hydrogen bond interactions between each oxyluciferin derivative and the explicit water molecules by MD simulations. ii) Absorption spectrum simulation, comparison with the experimental data and analysis of the involved electronic transitions and iii) Simulation of the emission spectrum and comparison with the experimental steady-state and time resolved data to get insight into the chemical nature of the emitter specie.

\section{Water/oxyluciferin hydrogen bond interactions.}

Before starting with the study of the photochemical properties of oxyluciferin and its analogues it is important to analyze the hydrogen bond patterning between the water molecules and each solute due to its known influence on both the absorption and emission spectra. ${ }^{40,47,61}$

Starting with the natural forms of oxyluciferin, we have calculated the average number of hydrogen bond interactions between the water molecules and the heteroatoms, $(\mathrm{O} 10, \mathrm{O} 11, \mathrm{~N} 3, \mathrm{~N} 3$ ' in Figure 1) along the MD trajectory (Table S1). By analyzing the data, general patterns can be set: $i$ ) for both the phenolate (Figure 1A and B) and enolate (Figure 1C) derivatives, the deprotonated oxygen leads three to four hydrogen bonds with three/four different water molecules, ii) regarding to the 
keto group (Figure 1A), between one and two water molecules interact with the oxygen atom, both in the phenol and in the phenolate derivatives, whereas iii) the interaction of only one water molecule with the oxygen of the enol group (Figure 1B) or phenol group (Figure 1C) has been observed during half of the simulation time and $i v$ ) the two nitrogen atoms of the structure present in general one hydrogen bond interaction in less than half of the simulation time. Moreover, we have observed that during the simulation time there is a continuous interchange of the water molecules involved in the hydrogen bond interactions with the ones of the solvation sphere, as could be expected at room temperature.

A)
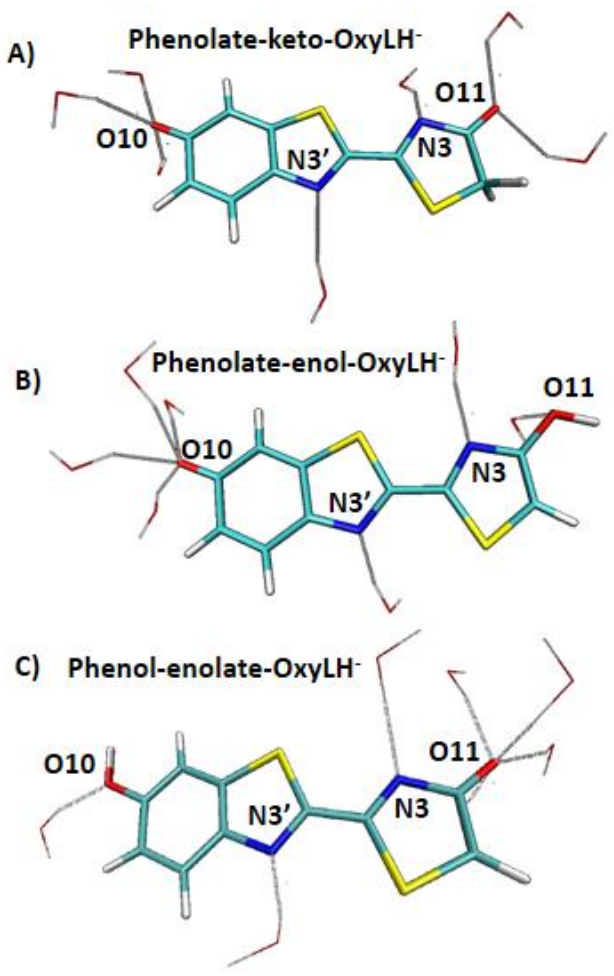

Figure 1. Graphical representation of the hydrogen bond pattern for selected MD snapshots of A) phenolate-keto-OxyLH; , B) phenolate-enol-OxyLH- and C) phenol-enolate-OxyLH'. Atom labelling for oxyluciferin heteroatoms is depicted. 
Similar hydrogen bond patterns have been found for the oxyluciferin analogues (Table S1). That is, neither the methyl nor the cyclopropyl substituents have a great influence on the hydrogen bond interactions. Just a slight difference was observed for phenolate-4-MeOxyL and enolate-6'$\mathrm{MeOxyL}^{-}$compared to their respective natural forms phenolate-enol-OxyLH${ }^{-}$and phenol-enolate$\mathrm{OxyLH}^{-}$. In these cases, the average number of hydrogen bonds found for the oxygen of the methoxy group is a bit larger than the one found for the corresponding natural forms, probably due to the inductive effect of the methyl group that increases the electronic charge of the oxygen atom.

\section{Absorption Spectra Simulation.}

Before starting the analysis of the absorption spectra and the electronic nature of the optically bright electronic transitions, it is essential to validate the procedure used to simulate the absorption spectra (see Methods section for details). For this aim, a comparison between the simulated and the experimental absorption spectra of the oxyluciferin analogues has been performed. We selected oxyluciferin analogues instead of the natural forms for this purpose as it is impossible to record experimentally the absorption spectra of each natural form in water due to the low thermal stability of some of them and, certainly, a mixture of different forms in equilibrium (Chart 1). ${ }^{33,34}$ Hence, we simulated the absorption spectra for the protonated and deprotonated forms of 5,5-CprOxyLH, 4-MeOxyLH and 6'-MeOxyLH. By analyzing Figure 2, we can conclude that a good overall agreement between the simulated and experimental spectra has been achieved in terms of both the shape and the maximum absorption wavelengths. 
A)

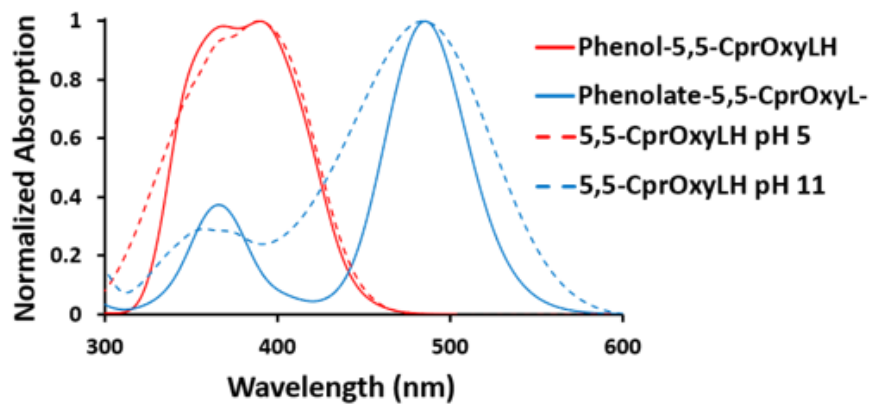

B)

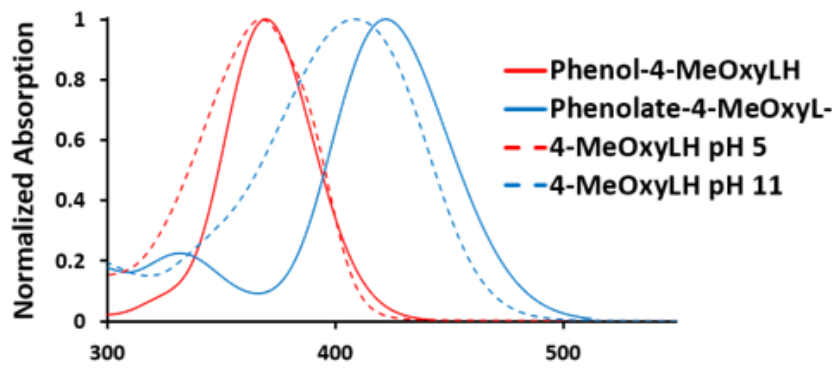

C)

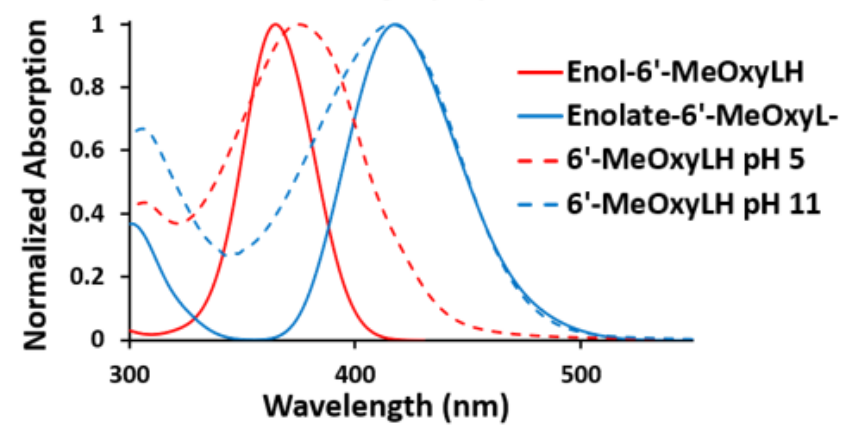

Figure 2. Normalized simulated absorption spectra (thick lines) of both the protonated (red lines) and deprotonated (blue lines) forms and the normalized experimental absorption spectra (dotted lines) recorded at pH 5 (red lines) and pH 11 (blue lines) for A) 5,5-CprOxyLH, B) 4-MeOxyLH and C) 6'MeOxyLH.

After the validation procedure, we have performed a systematic comparison between the simulated absorption spectra of the oxyluciferin analogues and their corresponding natural forms to give insight into their suitability to mimic oxyluciferin in water. In general, almost overlapped absorption spectra have been obtained (Figure S4). Only for phenol-5,5-CprOxyLH, the structured shape of the dominating absorption band is slightly different to the one simulated for phenol-keto- 
$\mathrm{OxyLH}_{2}$ but both share the same specificity compared to other chemical forms: absorption bands are significantly broader and more structured. Hence, we can conclude that, the electronic structures and absorption spectra of the proposed oxyluciferin analogues resemble to a great extent the ones of the natural forms in water. Moreover, we show that the introduction of the methyl and cyclopropyl groups do not modify the shape nor the maximum absorption wavelength of the absorption spectra.

In addition to the overall spectral shapes, we may analyze the electronic nature of the corresponding optically bright transitions. Starting with the keto derivatives, both phenol-keto$\mathrm{OxyLH}_{2}$ and phenol-5,5-CprOxyLH present one of the broadest absorption bands (red line in Figure $2 \mathrm{~A})$. The computational data predict that the first three singlet excited states $\left(\mathrm{S}_{1}, \mathrm{~S}_{2}\right.$ and $\left.\mathrm{S}_{3}\right)$ are optically bright and close in energy such that the dominating low-energy absorption band actually corresponds to three different electronic transitions, explaining the width and structured shape of both the experimental and simulated spectra (Figure 3A and Figure S4C). To analyze the electronic nature of these three transitions, we have investigated the molecular orbitals involved in each one. The electronic nature of the $\mathrm{S}_{0}-\mathrm{S}_{1}$ transition corresponds to the previously reported transition responsible of oxyluciferin emission ${ }^{28,36,62,63}$ for which the electron density of the HOMO is mainly located on the benzothiazole moiety and the LUMO on the thiazolone part. The $\mathrm{S}_{0}-\mathrm{S}_{2}$ transition is similar to the $\mathrm{S}_{0}-\mathrm{S}_{1}$ transition but part of the electron density of the bonding orbital (HOMO-1) is located on the sulfur atom of the benzothiazole moiety. As a consequence of the close energy between $S_{1}$ and $S_{2}$, their electronic nature is largely mixed which can explain their similar absorption intensities as has been shown by computing their oscillator and transition dipole moment strengths (see Supporting Information and Figure S9). On the other hand, the $\mathrm{S}_{0}-\mathrm{S}_{3}$ transition is the most different one as it involves a local rearrangement of the electron density on 
the thiazolone part (Figure 3A). A similar picture has been observed for phenol-keto-OxyLH 2 (Figure S5D).
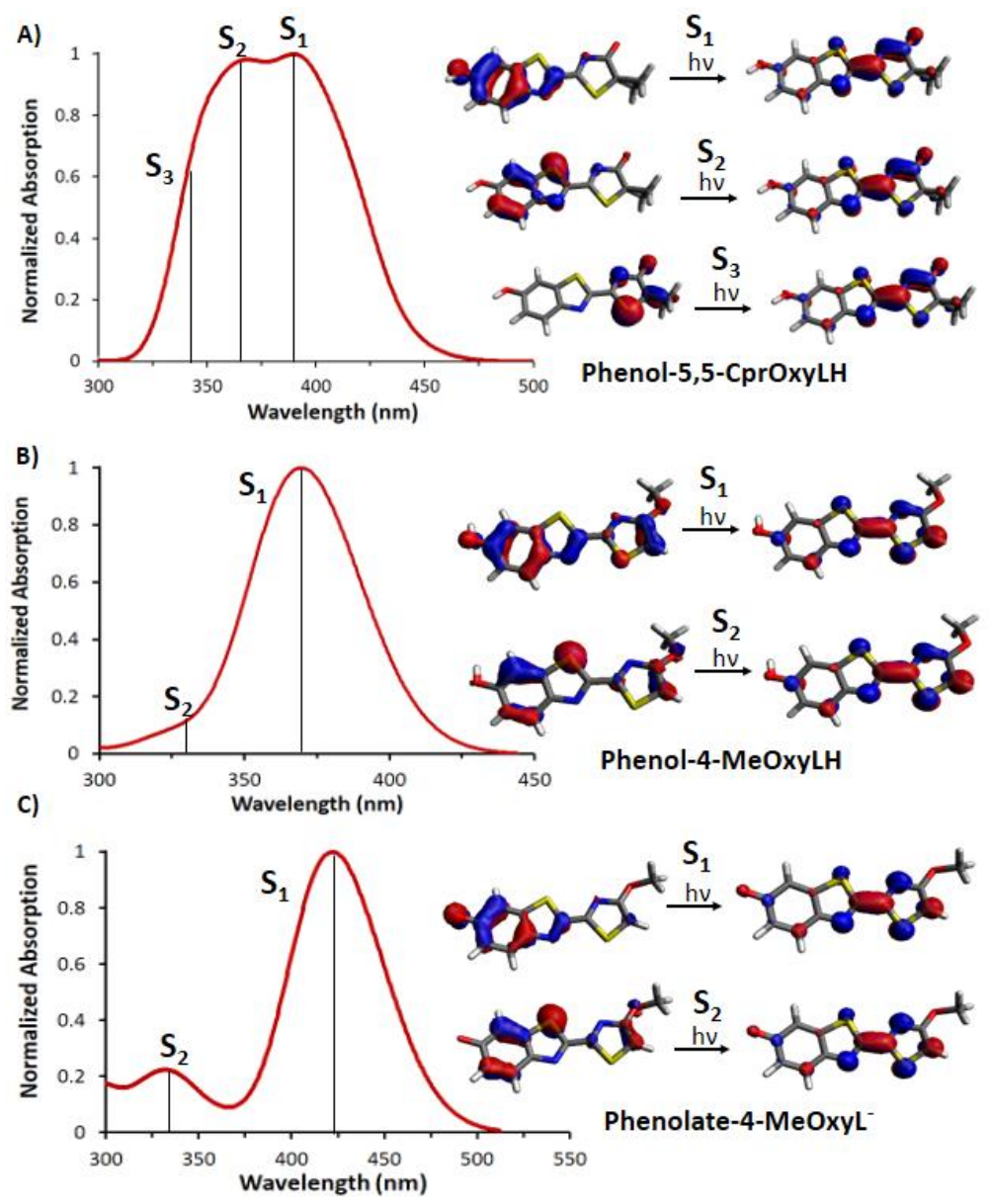

Figure 3. Simulated absorption spectra and molecular orbitals involved in each optically bright electronic transition of A) phenol-5,5-CprOxyLH, B) phenol-4-MeOxyLH and C) phenolate-4-MeOxyL:

On the contrary, a simplest picture is observed for the corresponding deprotonated compounds phenolate-keto-OxyLH ${ }^{-}$and phenolate-5,5-CprOxyL-: the $\mathrm{S}_{0}-\mathrm{S}_{1}$ and $\mathrm{S}_{0}-\mathrm{S}_{2}$ transitions lead to two well-separated absorption bands (Figure 2A, S4D and S5A). Their electronic nature is similar to the ones found for the corresponding protonated derivatives. 
A similar study has been done for phenol-enol- $\mathrm{OxyLH}_{2}$ and its two corresponding analogues phenol-4-MeOxyLH and enol-6'-MeOxyLH. Experimentally, the low-energy absorption band is similarly broad (red lines in Figure 2B and 2C) but in this case no strong shoulders are observed like for the phenol-keto derivatives, although the band is slightly asymmetric. We predict that this band mostly corresponds to the $\mathrm{S}_{0}-\mathrm{S}_{1}$ transition, while the less intense $\mathrm{S}_{0}-\mathrm{S}_{2}$ transition is hidden in the tail (Figure 3B, S4A, S4E and S5B). In this case, the oscillator strength found for $\mathrm{S}_{2}$ is one order of magnitude smaller than the one of $S_{1}(0.5$ vs. 6 a.u), in contrast to the similar intensity found for these two transitions for the phenol-keto derivatives, as both states are quite far in energy and their electronic nature is not mixed (Figure S9). Hence, we can state that the general picture found for the phenol-enol derivatives is simpler than the one of the phenol-keto ones, since the $\mathrm{S}_{1}$ and $\mathrm{S}_{2}$ states are energetically more distant and $\mathrm{S}_{2}$ is not so intense. The electronic nature of $\mathrm{S}_{0}-\mathrm{S}_{1}$ and $\mathrm{S}_{0}-\mathrm{S}_{2}$ transitions are similar to the ones found for the phenol-keto derivatives.

Regarding to the deprotonated forms, the picture of phenolate-enol-OxyLH${ }^{-}$and phenolate-4$\mathrm{MeOxyL}^{-}$is very similar to the one found for their protonated forms: a broad band with the $\mathrm{S}_{0}-\mathrm{S}_{2}$ transition hidden in the tail (Figure 2B and S4B). For phenol-enolate-OxyLH $\mathrm{H}^{-}$and enolate-6'MeOxyL ${ }^{-}$the $\mathrm{S}_{1}$ and $\mathrm{S}_{2}$ states are even more distant in energy yielding to two well-separated bands. (Figure 2C, S4F and S5C).

Hence, thanks to the computation of the vertical transitions we have been able to rationalize the different absorption band shapes found for oxyluciferin natural forms and its analogues and what is more, reveal hidden transitions which were experimentally embedded in high intense bands. Moreover, we have shown that the studied oxyluciferin analogues present almost identical absorption spectra and electronic nature of the vertical transitions than the corresponding natural forms in water (Figure 4A and B). It should be recalled that for the analogues under study, a quite 
good agreement between experimental and simulated absorption spectra has been achieved (Figure 2). In addition, the simulated absorption spectra of oxyluciferin natural forms in water are in line with the reported ones obtained by applying the multivariate curve resolution-alternating leastsquares (MCR-ALS) procedure (Figure 4B and C). ${ }^{34}$
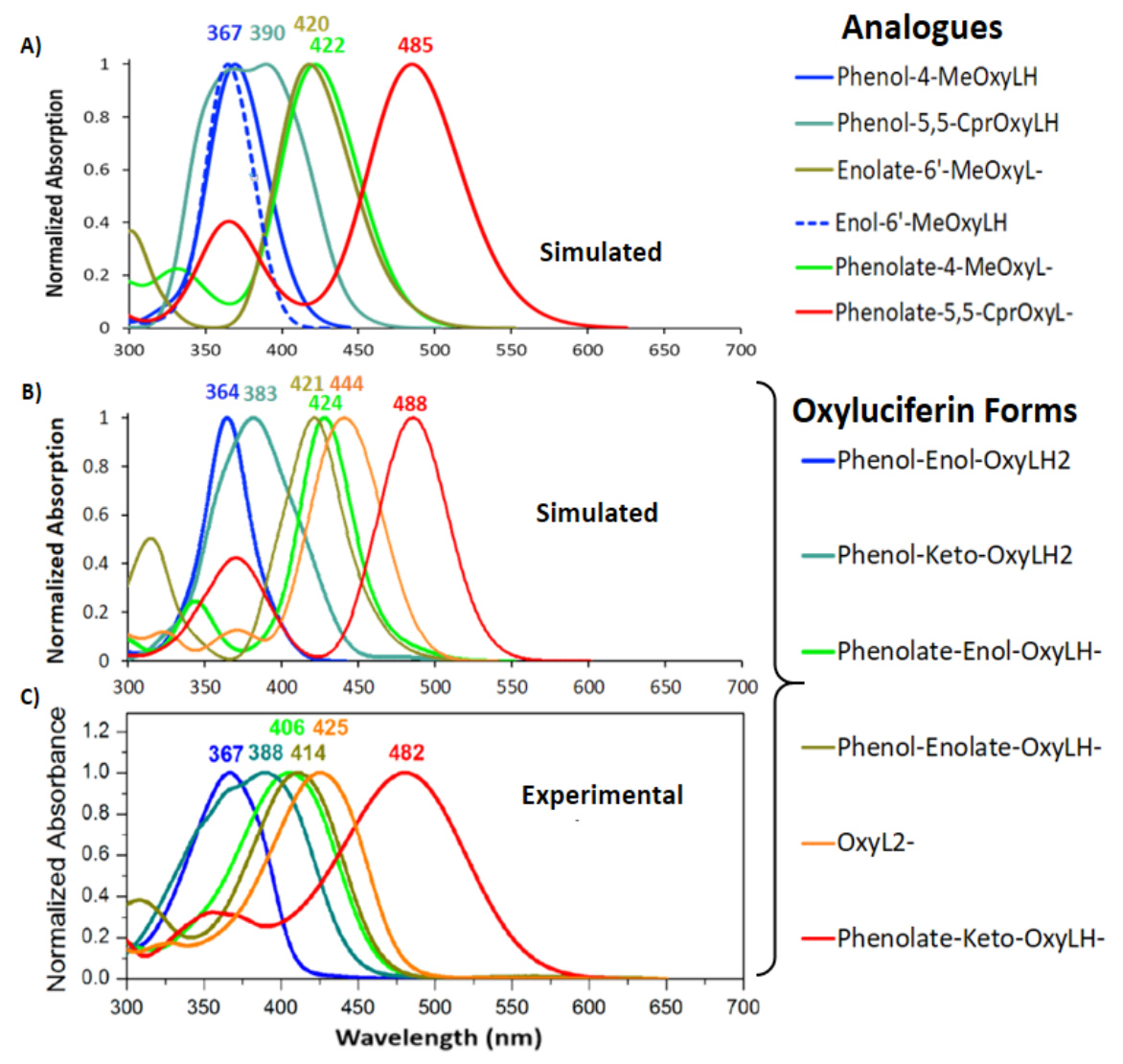

Figure 4. Summary graph of the simulated absorption spectra considering explicit water molecules of A) oxyluciferin analogues and B) natural oxyluciferin forms. C) Absorption spectra obtained by the MCRALS procedure ${ }^{34}$ for the natural oxyluciferin forms (Reprinted with permission from Ghose, A.; Rebarz, M.; Maltsev, O. V.; Hintermann, L.; Ruckebusch, C.; Fron, E.; Hofkens, J.; Mély, Y.; Naumov, P.; Sliwa, M.; Didier, P. J. Phys. Chem. B 2015, 119 (6), 2638-2649. Copyright 2017 American Chemical Society). 


\section{Emission Spectra Simulation.}

In this section we analyzed and rationalized the shape of the emission spectra of the three selected oxyluciferin analogues and their corresponding natural forms (Chart 2) in both their protonated and deprotonated forms. With this aim, a combined experimental and computational study has been performed.

Starting with the experimental results, the steady-state emission spectra recorded at $\mathrm{pH} 5$ and 11 are quite similar for all oxyluciferin analogues (dotted red and blue lines in Figure 5). In particular for 5,5-CprOxyLH and 6'-MeOxyLH the spectra at the two $\mathrm{pH}$ overlap while for 4-MeOxyLH only an additional weak, higher-energy band arises at $\mathrm{pH} 5$ (Figure 5B). This is due to the fact that the protonated species are very short-lived in the excited state due to Excited State Proton Transfer (ESPT) and hence, essentially only the emission of the deprotonated forms is observed by steadystate fluorescence, even if the protonated form is excited. To evidence the ultrafast ESPT, femtosecond pump-probe measurements were performed on 5,5-CprOxyLH in two different $\mathrm{pH}$ conditions: the compound was dissolved in aqueous buffer at $\mathrm{pH}=5$, where it is protonated, and at $\mathrm{pH}=11$ where it is deprotonated (pKa 7.8). 


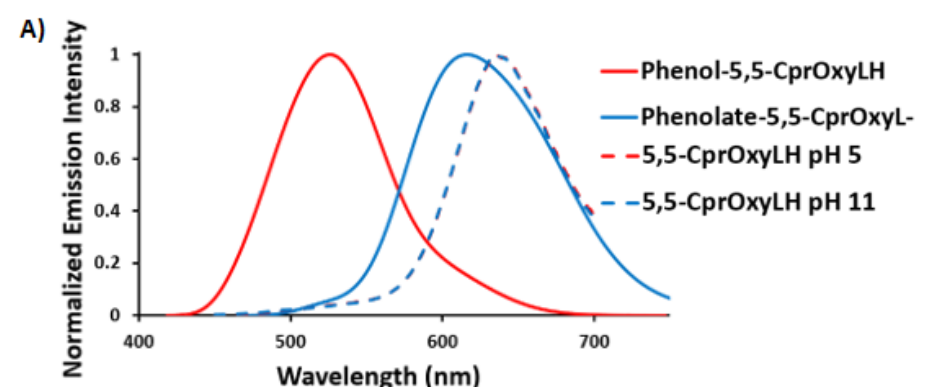

B)
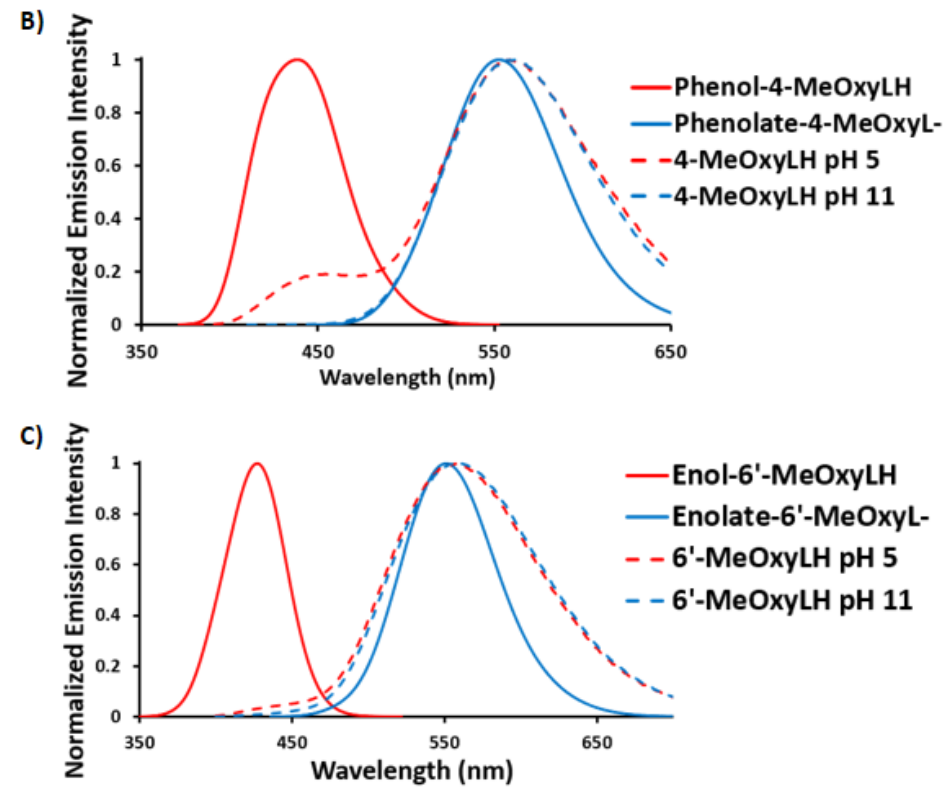

Figure 5. Normalized simulated emission spectra (thick lines) of both the protonated (red lines) and deprotonated (blue lines) forms and the normalized experimental emission spectra (dotted lines) recorded at pH 5 (red lines) and pH 11 (blue lines) of A) 5,5-CprOxyLH, B) 4-MeOxyLH and C) 6'-MeOxyLH.

To extract the characteristic time associated to the proton transfer we analyzed the positive contribution associated to the stimulated emission (SE) of the protonated and the deprotonated forms for both $\mathrm{pH}$. At $\mathrm{pH}=11$, the compound is already deprotonated in the ground state. The positive band around $610 \mathrm{~nm}$ rapidly red shifts to $640 \mathrm{~nm}$ that is the fluorescence emission wavelength of the deprotonated compound (see Figure 6). Hence the $640 \mathrm{~nm}$ band is attributed to SE from the $\mathrm{S}_{1}$ state, and its early red shift is attributed to the rapid Stokes shift due to vibrational and/or solvent relaxation ( $<1 \mathrm{ps}$ ), according to the fit of the $640 \mathrm{~nm}$ kinetic trace (Figure 6). After 
this initial rapid Stokes shift, the signal decays with a monoexponential behavior on the $700 \mathrm{ps}$ time scale in excellent agreement with the measured fluorescence lifetime. ${ }^{34} \mathrm{At} \mathrm{pH}=5$, the early band centered at $525 \mathrm{~nm}$ is interpreted as SE, in agreement with the known $525 \mathrm{~nm}$ emission of the protonated species. ${ }^{34}$ This band rapidly decays while the $640 \mathrm{~nm}$ band characterizing the emission of the deprotonated form rises. This spectral evolution is a clear signature of the ESPT. The rise and decay of the $640 \mathrm{~nm}$ and $525 \mathrm{~nm}$ bands, respectively, are displayed in Figure 6 . Their global fit confirms that both kinetics evolve simultaneously and bi-exponentially with the same 1.5 ps and 16.5 ps time constants. Finally, after formation of the deprotonated form in the excited state, the latter decays on the same time scale as at $\mathrm{pH}=11$.
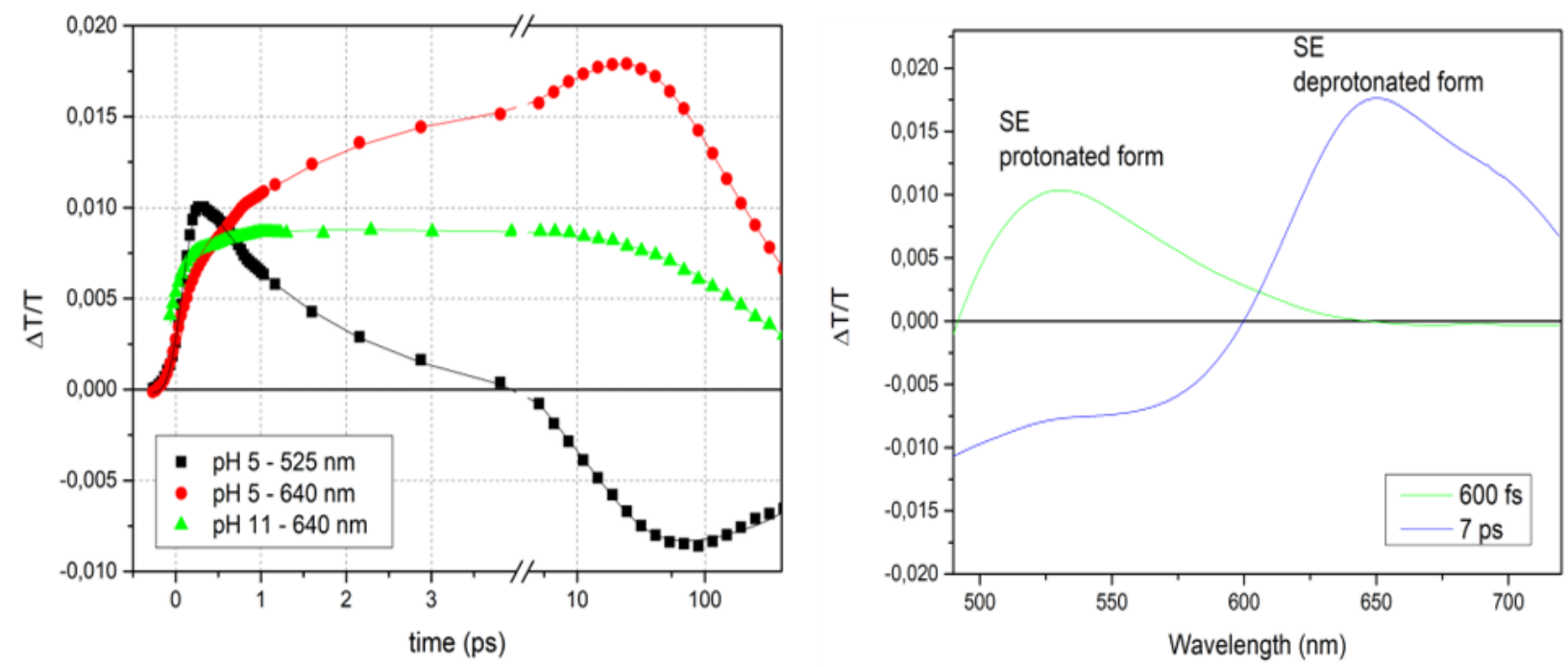

Figure 6. Left Panel: Results of a pump-probe experiment performed on 5,5-CprOxyLH in water at $\mathrm{pH}=11$ and $\mathrm{pH}=5$. Green symbols and line: kinetic trace of the $\mathrm{SE}$ at $640 \mathrm{~nm}$ for $\mathrm{pH}=11$ and its fit. Black symbols and line: $\mathrm{pH}=5$ kinetic traces at $525 \mathrm{~nm}$ attributed to the emission of protonated species and its fit. Red symbols and line: $\mathrm{pH}=5$ kinetic traces at $640 \mathrm{~nm}$ attributed to the emission of the deprotonated species and its fit. Right Panel: Differential transmission spectra of the probe beam measured at pH 5 for two pump-probe delays. 
These preliminary results demonstrate that the excited state dynamics of the 5,5-CprOxyLH is ruled by a very fast ESPT reaction. For this reason, the emission spectra recorded at $\mathrm{pH} 5$ and 11 by steady-state fluorescence are identical.

Next, we compared the emission wavelengths maxima of the three analogues previously measured by steady-state spectroscopy with the simulated spectra. ${ }^{34}$ For all the analogues, the simulated spectra of the deprotonated forms match the steady-state fluorescence spectra recorded at $\mathrm{pH} 11$ (thick and dotted blue lines in Figure 5) with maxima (620, 554 and $551 \mathrm{~nm}$ for 5,5-CprOxyLH, 4-MeOxyLH and 6'-MeOxyLH, respectively) in good agreement with the values recorded by steady-state fluorescence $(640,560$ and $550 \mathrm{~nm}$ for 5,5-CprOxyLH, 4-MeOxyLH and 6'MeOxyLH, respectively) ${ }^{34}$ Regarding the simulated emission spectra of the protonated forms (thick red lines in Figure 5), they cannot be directly compared, for all compounds, with the steadystate fluorescence spectra recorded at $\mathrm{pH} 5$ due to efficient ESPT, as demonstrated for 5,5CprOxyLH by pump probe experiments. In the case of 4-MeOxyLH, time-correlated single photon counting measurements evidenced a slower ESPT dynamics $(240 \mathrm{ps})^{34}$, which is slow enough to enable monitoring the steady-state emission from the protonated form (Figure 5B, red dotted line band centered at $450 \mathrm{~nm}$ ). Moreover, it was possible to experimentally determine the emission maxima of two analogues of the (protonated) phenol-enol and phenol-keto forms (6'-Me-5,5CprOxyL and 4,6'-DMeOxyL, where no ESPT reaction can occur due to replacement of the labile $\mathrm{H}$ atom by a methyl group) at 525 and $445 \mathrm{~nm}$, respectively. ${ }^{34}$ The simulated emission maxima of 5,5-CprOxyLH, 4-MeOxyLH and 6'-MeOxyLH, are obtained at 526, 439 and $430 \mathrm{~nm}$, respectively thus fully in line with the experimental spectra.

Besides, we have also simulated the emission spectra of the six possible forms of oxyluciferin and compared with the ones simulated for the analogues (Figure S8). In all cases, almost overlapping 
spectra have been obtained, with less than $0.1 \mathrm{eV}$ differences in the emission maxima. Hence, we can state that all the analogues studied in this work are representative of oxyluciferin in water in terms of the emission properties.

So, thanks to this analysis we have been able to rationalize the emission spectra experimentally recorded for the three analogues at $\mathrm{pH} 5$ and 11, demonstrating an efficient ESPT for their protonated forms. Moreover, we have provided by simulation the emission spectra of the protonated forms of 5,5-CprOxyLH and 6'-MeOxyLH, impossible to record by steady-state experiments. Finally, we have shown that the analogues under study are representative of oxyluciferin natural forms in water (Figure 7A and B). It should be remarked that it has been achieved a good agreement between the spectra obtained by applying the MCR-ALS procedure ${ }^{34}$ and the simulated emission spectra of the six possible forms of Oxyluciferin (Figure 7B and C).

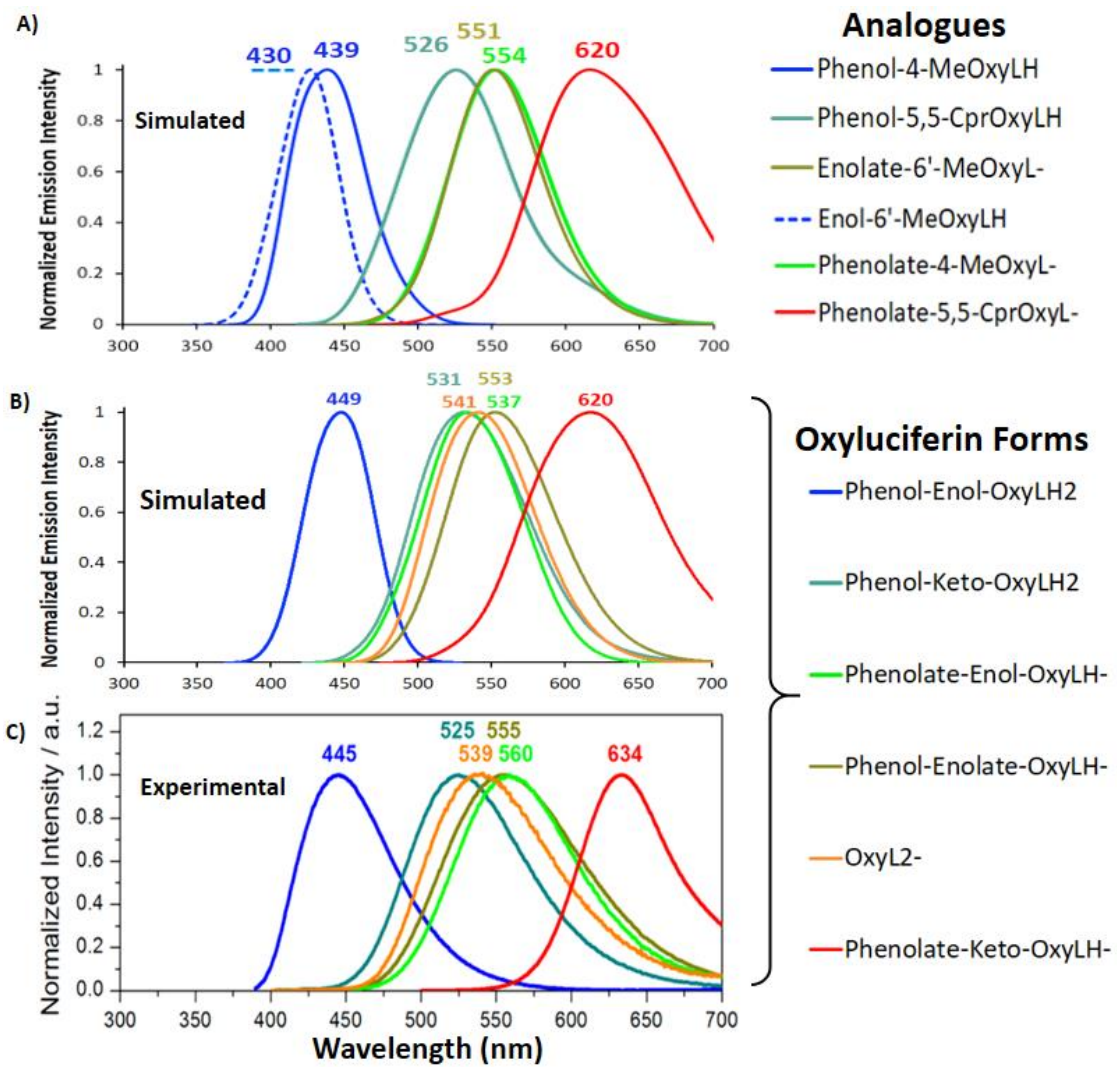


Figure 7. Summary graph of the simulated emission spectra considering explicit water molecules of A) oxyluciferin analogues and B) natural oxyluciferin forms. C) Emission spectra obtained by the MCR-ALS procedure $^{34}$ of natural oxyluciferin forms. (Reprinted with permission from Ghose, A.; Rebarz, M.; Maltsev, O. V.; Hintermann, L.; Ruckebusch, C.; Fron, E.; Hofkens, J.; Mély, Y.; Naumov, P.; Sliwa, M.; Didier, P. J. Phys. Chem. B 2015, 119 (6), 2638-2649. Copyright 2017 American Chemical Society).

\section{CONCLUSIONS}

In this study, we have presented a simple procedure to simulate the absorption and emission spectra of oxyluciferin and their analogues in water solution. It has to be noted that this procedure considers at the same time the interaction of the solute with explicit water molecules and dynamical effects, giving a more realistic picture of the system. In general, a quite good agreement between the simulated and experimental spectra has been obtained hence, independently confirming the MCR-ALS results for oxyluciferin in water.

By analyzing the electronic nature of the vertical transitions, we have found a main difference between phenol-enol and phenol-keto derivatives: the absorption band of the phenol-keto derivatives was found to be structured, since three optically bright excited states are close in energy; whereas for the phenol-enol derivatives an intense band corresponding to the $\mathrm{S}_{0}-\mathrm{S}_{1}$ transition has been found with a high-energy tail corresponding to $\mathrm{S}_{0}-\mathrm{S}_{2}$. Moreover, it has been shown the key importance of including the dynamical effect for phenol-keto derivatives to reproduce the structured absorption spectra experimentally found.

Regarding to the emission spectra, we have demonstrated an ultrafast ESPT for the neutral phenolketo form, not time-resolved in our previous work. ${ }^{34}$ In addition, we report the simulated emission spectra of the protonated forms, being impossible to record them by steady-state experiments. 
Finally, thanks to the comparative study of the photochemical properties of the natural oxyluciferin forms and their corresponding analogues presented in this work, we have demonstrated that these analogues can be used to mimic natural oxyluciferin forms, at least in water solution. This will promote future studies focused on rationalizing the emission color modulation in fireflies, as the possible chemical equilibria can be smartly blocked by using these derivatives.

\section{ASSOCIATED CONTENT}

Supporting Information. Computational Details. Absorption Spectrum Shape Convergence. Analysis of Hydrogen Bond Interactions. Simulated Absorption Spectra Optimizing the Chromophore. Simulated Absorption Spectra of Oxyluciferin Forms and Analogues. Analysis of the Absorption Spectra of Oxyluciferin Analogues. Simulated Emission Spectra Optimizing the Chromophore. Emission Spectrum Shape Convergence. Simulated Emission Spectra of Oxyluciferin Forms and Analogues. Emission Oscillator Strength. Relative Absorption Intensity for Phenol-keto and Phenol-enol Derivatives.

\section{AUTHOR INFORMATION}

\section{Corresponding Author}

*E-mail: cristina.garciairiepa@u-pem.fr

*E-mail: isabelle.navizet@u-pem.fr

\section{ACKNOWLEDGMENT}

All authors are grateful to the French Agence Nationale de la Recherche (grant ANR-BIOLUM ANR-16-CE29-0013). Cristina García-Iriepa acknowledge Fundación Ramón Areces for a postdoctoral fellowship. 


\section{REFERENCES}

(1) Shimomura, O. Bioluminescence : Chemical Principles and Methods; World Scientific: Singapore, 2012.

(2) Herring, P. J. Bioluminescence in Action; Academic Press: London New York, 1978.

(3) Zimmer, M. Green Fluorescent Protein (GFP): Applications, Structure, and Related Photophysical Behavior. Chem. Rev. 2002, 102, 759-782.

(4) Tsien, R. Y. The Green Fluorescent Protein. Annu. Rev. Biochem. 1998, 67 (1), 509-544.

(5) Remington, S. J. Green Fluorescent Protein: A Perspective. Protein Sci. 2011, 20 (9), 1509-1519.

(6) Baubet, V.; Le Mouellic, H.; Campbell, A. K.; Lucas-Meunier, E.; Fossier, P.; Brúlet, P. Chimeric Green Fluorescent Protein-Aequorin as Bioluminescent Ca2+ Reporters at the Single-Cell Level. Proc. Natl. Acad. Sci. U. S. A. 2000, 97 (13), 7260-7265.

(7) Fraga, H.; Allue, I.; Bowers, K.; Cobbold, P.; Southworth, T. L.; Hall, M. S.; Pangburn, S. J.; Buck, L. M.; Leach, F. R. Firefly Luminescence: A Historical Perspective and Recent Developments. Photochem. Photobiol. Sci. 2008, 7 (2), 146.

(8) Kaskova, Z. M.; Tsarkova, A. S.; Yampolsky, I. V. 1001 Lights: Luciferins, Luciferases, Their Mechanisms of Action and Applications in Chemical Analysis, Biology and Medicine. Chem. Soc. Rev. 2016, 45 (21), 6048-6077.

(9) Griffiths, M. The Role of ATP Bioluminescence in the Food Industry: New Light on Old Problems. Food Technol. 1996, 50, 62-73.

(10) Shinde, R.; Perkins, J.; Contag, C. H. Luciferin Derivatives for Enhanced in Vitro and in Vivo Bioluminescence Assays. Biochemistry 2006, 45, 11103-11112.

(11) Greer, L. F.; Szalay, A. A. Imaging of Light Emission from the Expression of Luciferases in Living Cells and Organisms: A Review. Luminescence 2002, 17 (1), 43-74.

(12) Kricka, L. J. Application of Bioluminescence and Chemiluminescence in Biomedical Sciences. Methods Enzymol. 2000, 305, 333-345.

(13) Ohkuma, H.; Abe, K.; Kosaka, Y.; Maeda, M. Detection of Luciferase Having Two Kinds of Luminescent Colour Based on Optical Filter Procedure: Application to an Enzyme Immunoassay. Luminescence 2000, 15 (1), 21-27.

(14) Shao, Q.; Jiang, T.; Ren, G.; Cheng, Z.; Xing, B. Photoactivable Bioluminescent Probes for Imaging Luciferase Activity. Chem. Commun. 2009, 0 (27), 4028.

(15) Doyle, T. C.; Burns, S. M.; Contag, C. H. In Vivo Bioluminescence Imaging for Integrated Studies of Infection. Cell. Microbiol. 2004, 6 (4), 303-317.

(16) Fan, F.; Wood, K. V. Bioluminescent Assays for High-Throughput Screening. Assay Drug Dev. Technol. 2007, 5 (1), 127-136.

(17) White, E. H.; Rapaport, E.; Seliger, H. H.; Hopkins, T. A. The Chemi- and Bioluminescence of Firefly Luciferin: An Efficient Chemical Production of Electronically Excited States. Bioorg. Chem. 1971, 1 (1-2), 92-122.

(18) Deluca, M. Firefly Luciferase. Adv. Enzymol. Relat. Areas Mol. Biol. 1976, 44, 37-68.

(19) Navizet, I.; Liu, Y.-J.; Ferré, N.; Roca-Sanjuán, D.; Lindh, R. The Chemistry of Bioluminescence: An Analysis of Chemical Functionalities. ChemPhysChem 2011, 12 (17), 3064-3076.

(20) Ando, Y.; Niwa, K.; Yamada, N.; Enomoto, T.; Irie, T.; Kubota, H.; Ohmiya, Y.; Akiyama, H. Firefly Bioluminescence Quantum Yield and Colour Change by pH-Sensitive Green Emission. Nat. Photonics 2008, 2 (1), 44-47.

(21) Wang, Y.; Kubota, H.; Yamada, N.; Irie, T.; Akiyama, H. Quantum Yields and Quantitative Spectra of Firefly Bioluminescence with Various Bivalent Metal Ions. Photochem. Photobiol. 2011, 87 (4), 846-852.

(22) Mochizuki, T.; Wang, Y.; Hiyama, M.; Akiyama, H. Robust Red-Emission Spectra and Yields in Firefly Bioluminescence against Temperature Changes. Appl. Phys. Lett. 2014, 104 (21), 213704.

(23) Wang, Y.; Akiyama, H.; Terakado, K.; Nakatsu, T. Impact of Site-Directed Mutant Luciferase on Quantitative Green and Orange/red Emission Intensities in Firefly Bioluminescence. Sci. Rep. 2013, 
3, 2490.

(24) Mofford, D. M.; Reddy, G. R.; Miller, S. C. Aminoluciferins Extend Firefly Luciferase Bioluminescence into the Near-Infrared and Can Be Preferred Substrates over D -Luciferin. J. Am. Chem. Soc. 2014, 136 (38), 13277-13282.

(25) Reddy, G. R.; Thompson, W. C.; Miller, S. C. Robust Light Emission from Cyclic Alkylaminoluciferin Substrates for Firefly Luciferase. J. Am. Chem. Soc. 2010, 132 (39), 1358613587.

(26) Hosseinkhani, S. Molecular Enigma of Multicolor Bioluminescence of Firefly Luciferase. Cell. Mol. Life Sci. 2011, 68 (7), 1167-1182.

(27) Hirano, T.; Hasumi, Y.; Ohtsuka, K.; Maki, S.; Niwa, H.; Yamaji, M.; Hashizume, D. Spectroscopic Studies of the Light-Color Modulation Mechanism of Firefly (Beetle) Bioluminescence. J. Am. Chem. Soc. 2009, 131 (6), 2385-2396.

(28) Navizet, I.; Liu, Y.-J.; Ferré, N.; Xiao, H.-Y.; Fang, W.-H.; Lindh, R. Color-Tuning Mechanism of Firefly Investigated by Multi-Configurational Perturbation Method. J. Am. Chem. Soc. 2010, 132 (2), 706-712.

(29) Naumov, P.; Kochunnoonny, M. Spectral-Structural Effects of the Keto-Enol-Enolate and Phenol-Phenolate Equilibria of Oxyluciferin. J. Am. Chem. Soc. 2010, 132 (33), 11566-11579.

(30) Maltsev, O. V.; Nath, N. K.; Naumov, P.; Hintermann, L. Why Is Firefly Oxyluciferin a Notoriously Labile Substance? Angew. Chemie Int. Ed. 2014, 53 (3), 847-850.

(31) Naumov, P.; Ozawa, Y.; Ohkubo, K.; Fukuzumi, S. Structure and Spectroscopy of Oxyluciferin, the Light Emitter of the Firefly Bioluminescence. J. Am. Chem. Soc. 2009, 131 (32), 11590-11605.

(32) Branchini, B. R.; Martha H. Murtiashaw, Rachelle A. Magyar, Nathan C. Portier, M. C. R.; Stroh, J. G. Yellow-Green and Red Firefly Bioluminescence from 5,5-Dimethyloxyluciferin. J. Am. Chem. Soc. 2002, 124, 2112-2113.

(33) Rebarz, M.; Kukovec, B.-M.; Maltsev, O. V.; Ruckebusch, C.; Hintermann, L.; Naumov, P.; Sliwa, M. Deciphering the Protonation and Tautomeric Equilibria of Firefly Oxyluciferin by Molecular Engineering and Multivariate Curve Resolution. Chem. Sci. 2013, 4 (10), 3803-3809.

(34) Ghose, A.; Rebarz, M.; Maltsev, O. V.; Hintermann, L.; Ruckebusch, C.; Fron, E.; Hofkens, J.; Mély, Y.; Naumov, P.; Sliwa, M.; Didier, P. Emission Properties of Oxyluciferin and Its Derivatives in Water: Revealing the Nature of the Emissive Species in Firefly Bioluminescence. J. Phys. Chem. $B$ 2015, 119 (6), 2638-2649.

(35) Ghose, A.; Maltsev, O. V.; Humbert, N.; Hintermann, L.; Arntz, Y.; Naumov, P.; Mély, Y.; Didier, P. Oxyluciferin Derivatives: A Toolbox of Environment-Sensitive Fluorescence Probes for Molecular and Cellular Applications. J. Phys. Chem. B 2017, 121 (7), 1566-1575.

(36) Galina Orlova, J. D. G.; Brovko, L. Y. Theoretical Study of the Amazing Firefly Bioluminescence: The Formation and Structures of the Light Emitters. J. Am. Chem. Soc. 2003, 125, 6962-6971.

(37) Navizet, I.; Roca-Sanjuán, D.; Yue, L.; Liu, Y.-J.; Ferré, N.; Lindh, R. Are the Bio- and Chemiluminescence States of the Firefly Oxyluciferin the Same as the Fluorescence State? Photochem. Photobiol. 2013, 89 (2), 319-325.

(38) Hiyama, M.; Akiyama, H.; Koga, N. Theoretical Insights into the Effect of $\mathrm{pH}$ Values on Oxidation Processes in the Emission of Firefly Luciferin in Aqueous Solution. Luminescence 2017, 32 (6), 1100-1108.

(39) Hiyama, M.; Akiyama, H.; Mochizuki, T.; Yamada, K.; Koga, N. Analysis of Photoexcitation Energy Dependence in the Photoluminescence of Firefly Luciferin. Photochem. Photobiol. 2014, 90 (4), n/a-n/a.

(40) Noguchi, Y.; Hiyama, M.; Shiga, M.; Sugino, O.; Akiyama, H. Reverse Stability of Oxyluciferin Isomers in Aqueous Solutions. J. Phys. Chem. B 2016, 120 (34), 8776-8783.

(41) Hiyama, M.; Akiyama, H.; Yamada, K.; Koga, N. Theoretical Study of Firefly Luciferin pKa Values-Relative Absorption Intensity in Aqueous Solutions. Photochem. Photobiol. 2013, 89 (3), 571-578.

(42) Hiyama, M.; Mochizuki, T.; Akiyama, H.; Koga, N. Analysis of Oxyluciferin Photoluminescence 
Pathways in Aqueous Solutions. Photochem. Photobiol. 2015, 91 (1), 74-83.

Yang, T.; Goddard, J. D. Predictions of the Geometries and Fluorescence Emission Energies of Oxyluciferins. J. Phys. Chem. A 2007, 111, 4489-4497.

(44) Hiyama, M.; Noguchi, Y.; Akiyama, H.; Yamada, K.; Koga, N. Vibronic Structures in Absorption and Fluorescence Spectra of Firefly Oxyluciferin in Aqueous Solutions. Photochem. Photobiol. 2015, 91 (4), 819-827.

(45) Coccia, E.; Varsano, D.; Guidoni, L. Theoretical S1 $\leftarrow$ S0 Absorption Energies of the Anionic Forms of Oxyluciferin by Variational Monte Carlo and Many-Body Green's Function Theory. J. Chem. Theory Comput. 2017, 13 (9), 4357-4367.

(46) Falklöf, O.; Durbeej, B. Distinguishing between Keto-Enol and Acid-Base Forms of Firefly Oxyluciferin through Calculation of Excited-State Equilibrium Constants. J. Comput. Chem. 2014, 35 (30), 2184-2194.

(47) Hiyama, M.; Shiga, M.; Koga, N.; Sugino, O.; Akiyama, H.; Noguchi, Y. The Effect of Dynamical Fluctuations of Hydration Structures on the Absorption Spectra of Oxyluciferin Anions in an Aqueous Solution. Phys. Chem. Chem. Phys. 2017, 19 (15), 10028-10035.

(48) D.A. Case, V. Babin, J.T. Berryman, R.M. Betz, Q. Cai, D.S. Cerutti, T.E. Cheatham, III, T.A. Darden, R. E.; Duke, H. Gohlke, A.W. Goetz, S. Gusarov, N. Homeyer, P. Janowski, J. Kaus, I. Kolossváry, A. K.; T.S. Lee, S. LeGrand, T. Luchko, R. Luo, B. Madej, K.M. Merz, F. Paesani, D.R. Roe, A. Roitberg, C. S.; R. Salomon-Ferrer, G. Seabra, C.L. Simmerling, W. Smith, J. Swails, R.C. Walker, J. Wang, R.M. Wolf, X.; Kollman, W. and P. A. Amber14. University of California: San Francisco 2014.

(49) Jorgensen, W. L.; Chandrasekhar, J.; Madura, J. D.; Impey, R. W.; Klein, M. L. Comparison of Simple Potential Functions for Simulating Liquid Water. J. Chem. Phys. 1983, 79 (2), 926-935.

(50) Berendsen, H. J. C.; Postma, J. P. M.; Gunsteren, W. F. van; DiNola, A.; Haak, J. R. Molecular Dynamics with Coupling to an External Bath. J. Chem. Phys. 1984, 81, 3684.

(51) Becke, A. Becke's Three Parameter Hybrid Method Using the LYP Correlation Functional. J. Chem. Phys 1993, 98, 5648-5652.

(52) Stephens, P. J.; Devlin, F. J.; Chabalowski, C. F.; Frisch, M. J. Ab Initio Calculation of Vibrational Absorption and Circular Dichroism Spectra Using Density Functional Force Fields. J. Phys. Chem. 1994, 98, $11623-11627$.

(53) Lee, C.; Yang, W.; Parr, R. G. Development of the Colle-Salvetti Correlation-Energy Formula into a Functional of the Electron Density. Phys. Rev. B 1988, 37, 785-789.

(54) Berraud-Pache, R.; Navizet, I. QM/MM Calculations on a Newly Synthesised Oxyluciferin Substrate: New Insights into the Conformational Effect. Phys. Chem. Chem. Phys. 2016, 18 (39), 27460-27467.

(55) Min, C.-G.; Ren, A.-M.; Guo, J.-F.; Li, Z.-W.; Zou, L.-Y.; Goddard, J. D.; Feng, J.-K. A TimeDependent Density Functional Theory Investigation on the Origin of Red Chemiluminescence. ChemPhysChem 2010, 11 (1), 251-259.

(56) Min, C.-G.; Yan Leng; Yan-QinZhu; Xi-Kun Yang; Shao-Jun Huang; Ai-MinRen. Modification of Firefly Cyclic Amino Oxyluciferin Analogues Emitting Multicolor Light for OLED and nearInfrared Biological Window Light for Bioluminescence Imaging: A Theoretical Study. $J$. Photochem. Photobiol. A Chem. 2017, 336, 115-122.

(57) M. J. Frisch, G. W. Trucks, H. B. Schlegel, G. E. S.; M. A. Robb, J. R. Cheeseman, G. Scalmani, V. B.; B. Mennucci, G. A. Petersson, H. Nakatsuji, M. C.; X. Li, H. P. Hratchian, A. F. Izmaylov, J. Bloino, G. Z.; J. L. Sonnenberg, M. Hada, M. Ehara, K. Toyota, R. F.; J. Hasegawa, M. Ishida, T. Nakajima, Y. Honda, O. K.; H. Nakai, T. Vreven, J. A. Montgomery, Jr., J. E. P.; F. Ogliaro, M. Bearpark, J. J. Heyd, E. Brothers, K. N. K.; V. N. Staroverov, R. Kobayashi, J. Normand, K. R.; A. Rendell, J. C. Burant, S. S. Iyengar, J. Tomasi, M. C.; N. Rega, J. M. Millam, M. Klene, J. E. Knox, J. B. C.; V. Bakken, C. Adamo, J. Jaramillo, R. Gomperts, R. E.; Stratmann, O. Yazyev, A. J. Austin, R. Cammi, C. P.; J. W. Ochterski, R. L. Martin, K.Morokuma, V. G. Z.; G. A. Voth, P. Salvador, J. J. Dannenberg, S. Dapprich, A. D.; Daniels, O. Farkas, J. B. Foresman, J. V. Ortiz, J. C.; and D. J. 
Fox, and D. J. F. Gaussian 09. Gaussian, Inc.: Wallingford CT 2009.

(58) Ferré, N.; Ángyán, J. G. Approximate Electrostatic Interaction Operator for QM/MM Calculations. Chem. Phys. Lett. 2002, 356 (3-4), 331-339.

(59) Ponder, J. W. TINKER, Software Tools for Molecular Design, Version 6.3. Department of Biochemistry and Molecular Biophysics: Washington University School of Medicine, St. Louis, MO 2004.

(60) Melaccio, F.; Olivucci, M.; Lindh, R.; Ferré, N. Unique QM/MM Potential Energy Surface Exploration Using Microiterations. Int. J. Quantum Chem. 2011, 111 (13), 3339-3346.

(61) Ren, A.-M.; Goddard, J. D. Predictions of the Electronic Absorption and Emission Spectra of Luciferin and Oxyluciferins Including Solvation Effects. J. Photochem. Photobiol. B Biol. 2005, 81 (3), 163-170.

(62) Nakatani, N.; Hasegawa, J.; Nakatsuji, H. Red Light in Chemiluminescence and Yellow-Green Light in Bioluminescence: Color-Tuning Mechanism of Firefly, Photinus Pyralis, Studied by the Symmetry-Adapted Cluster-Configuration Interaction Method. J. Am. Chem. Soc. 2007, 129, 87568765.

(63) Milne, B. F. Red-Shifting the Optical Response of Firefly Oxyluciferin with Group 15/16 Substitutions. Phys. Chem. Chem. Phys. 2014, 16 (45), 24971-24977. 